\title{
Modelling of aluminium sheet forming at elevated temperatures
}

\author{
A.H. van den Boogaard* and J. Huétink* \\ ${ }^{*}$ Faculty of Engineering Technology, University of Twente, P.O. Box 217, 7500 AE Enschede, The Netherlands
}

\begin{abstract}
The formability of $\mathrm{Al}-\mathrm{Mg}$ sheet can be improved considerably, by increasing the temperature. By heating the sheet in areas with large shear strains, but cooling it on places where the risk of necking is high, the limiting drawing ratio can be increased to values above 2.5. At elevated temperatures, the mechanical response of the material becomes strain rate dependent. To accurately simulate warm forming of aluminium sheet, a material model is required that incorporates the temperature and strain-rate dependency. In this paper simulations are presented of the deep drawing of a cylindrical cup, using shell elements. It is demonstrated that the familiar quadratic Hill yield function is not capable of describing the plastic deformation of aluminium. Hardening can be described successfully with a physically based material model for temperatures up to $200^{\circ} \mathrm{C}$. At higher temperatures and very low strain rates, the flow curve deviates significantly from the model.
\end{abstract}

\section{INTRODUCTION}

In deep drawing of a cylindrical aluminium cup, the limiting drawing ratio can be increased considerably by controlling the temperature of different parts of the sheet $[1,2]$. By heating the flange up to $250^{\circ} \mathrm{C}$ and cooling the punch the limiting drawing ratio could be increased from 2.1 to 2.6 for a $5754-\mathrm{O}$ alloy [3, 4]. The optimal temperature distribution and punch velocity depend on the type of aluminium and the tool geometry. Because experience is lacking, computational analysis can assist in determination of the process window.

For a proper simulation of of the warm forming process a sufficiently accurate material model is needed. Focusing on the plastic deformation, the two most important building blocks of the material model are the yield function and the hardening model, including temperature and strain rate dependency. The applied material model is described next. With these models, a warm deep drawing experiment was simulated, which is presented thereafter.

\section{MATERIAL MODEL}

Material models for plastic deformation that are used in process simulations commonly apply a separation of the model in a yield surface and an evolution of the yield stress (hardening). The yield surface determines the plastic flow in a multiaxial stress state, while a hardening law determines the evolution of the yield surface. The same approach is used here.

\section{Vegter Yield Function}

The Vegter yield criterion $[5,6]$ defines a yield function for plane stress situations, directly based on experimental measurements on sheet material. The yield function is defined in the principal stress space. For planar anisotropic material, therefore, the yield function depends on the angle between the principal axes and the rolling direction. For a particular loading direction with respect to the rolling direction, four experiments are necessary to determine the model parameters: a pure shear test, a uniaxial tensile test, a plane strain tensile test and an equi-biaxial tensile test. Between the measured stress points a Bezier curve is used to describe the yield locus.

At yielding, not only the yield stress, but also the direction of plastic strain is determined. Based on Drucker's postulate, the normal to the yield locus has the same direction as the plastic strain rate. If the stress points and the yield locus directions are known, a set of Bezier curves can be constructed such that the ensuing yield locus is $C^{1}$ continuous. In the two-dimensional principal stress space, a stress point is represented by the vector $\vec{\sigma}=\left[\sigma_{1}, \sigma_{2}\right]^{\mathrm{T}}$. Every plane stress situation can now be represented by the principal stresses $\vec{\sigma}$ and the angle $\theta$ between the 1 st principal stress and the rolling direction.

For every part of the yield locus between two reference stress points, $\vec{\sigma}_{i}$ and $\vec{\sigma}_{j}$, a second order Bezier function is defined. The Bezier function is determined by the two reference stress points and the direction of the yield locus at the reference points, specified by $\rho=\dot{\varepsilon}_{2} / \dot{\varepsilon}_{1}$. The intersection of the two tangents at the reference points 


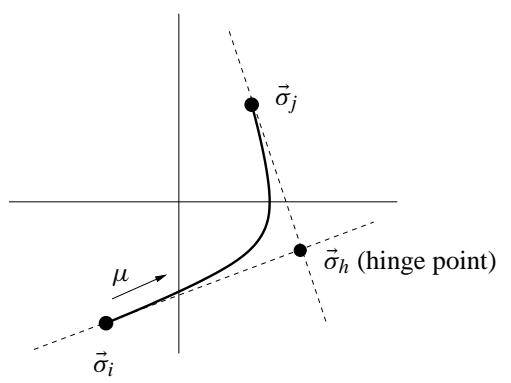

FIGURE 1. Second order Bezier curve between two reference stress points.

define the hinge point $\vec{\sigma}_{h}$, see Figure 1 . The yield locus between two reference stress points is defined by

$$
\vec{\sigma}_{\mathrm{loc}}=\vec{\sigma}_{i}+2 \mu\left(\vec{\sigma}_{h}-\vec{\sigma}_{i}\right)+\mu^{2}\left(\vec{\sigma}_{i}+\vec{\sigma}_{j}-2 \vec{\sigma}_{h}\right)
$$

with $\mu \in[0,1]$.

A yield function is constructed by defining an equivalent stress $\sigma_{\text {eq }}$, that is implied for any plane stress state by the relation

$$
\vec{\sigma}=\frac{\sigma_{\mathrm{eq}}}{\sigma_{\mathrm{f}}} \vec{\sigma}_{\mathrm{loc}}
$$

A function $\phi$ that is defined as

$$
\phi\left(\sigma, \varepsilon_{\mathrm{eq}}\right)=\sigma_{\mathrm{eq}}(\sigma)-\sigma_{\mathrm{f}}\left(\varepsilon_{\mathrm{eq}}\right)
$$

fulfils the condition that $\phi=0$ on the yield locus and $\phi<0$ in the elastic regime. The direction of the plastic strain rate can be calculated from the derivative of $\phi$ to the stress $\sigma$. Since $\phi$ is continuously differentiable, the plastic strain rate direction is continuous.

Planar anisotropic behaviour can be modelled by letting all reference stress points and corresponding normals depend on the angle $\theta$. The reference stress points and normals are defined by an interpolation, based on harmonic functions:

$$
\begin{aligned}
& \vec{\sigma}_{k}(\theta)=\sum_{j=0}^{n} \vec{\zeta}_{k j} \cos (2 j \theta) \\
& \rho_{k}(\theta)=\sum_{j=0}^{n} \varrho_{k j} \cos (2 j \theta)
\end{aligned}
$$

For common rolled materials, that develop four ears in deep drawing of a cylindrical cup, experiments are performed for the $0^{\circ}, 45^{\circ}$ and $90^{\circ}$ directions with respect to the rolling direction. For these materials a value $n=2$ is used. A complete yield locus for one specific angle $\theta$ is presented in Figure 2, including all reference and hinge points and the tangents.

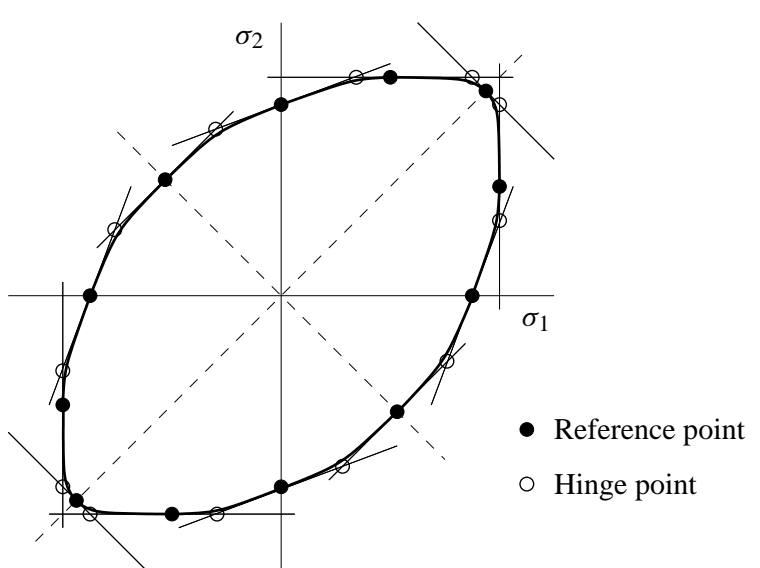

FIGURE 2. A complete Vegter yield locus, and tangents in the reference points.

\section{Flow Stress and Hardening}

The flow stress $\sigma_{\mathrm{f}}$, used in Equation (3), defines the resistance to plastic deformation of a material. Experiments show that above a temperature of approximately $100{ }^{\circ} \mathrm{C}$ the work hardening in $\mathrm{Al}-\mathrm{Mg}$ alloys depends on the temperature and strain rate.

\section{The Extended Nadai Model}

A typical phenomenological model is the Nadai or Swift relation. To add strain rate sensitivity to this relation, a power law is used:

$$
\sigma_{\mathrm{f}}=C(T)\left(\varepsilon+\varepsilon_{0}\right)^{n(T)} \dot{\varepsilon}^{m(T)}
$$

The parameters $C, n$ and $m$ can be made temperature dependent as demonstrated in [3].

\section{The Bergström Model}

The Bergström model starts with a decomposition of the flow stress into a strain and strain rate independent stress $\sigma_{0}$, a dynamic stress $\sigma^{*}$ that depends on the strain rate and temperature and a term $\sigma_{\mathrm{w}}$ that incorporates the work hardening:

$$
\sigma_{\mathrm{f}}=\sigma_{0}(T)+\sigma^{*}(\dot{\varepsilon}, T)+\sigma_{\mathrm{w}}(\rho, T)
$$

The influence of the dynamic stress $\sigma^{*}$ is decreasing with increasing temperature and is independent of the strain. In experiments with 5754-O alloy it is observed that the influence of the strain rate on the initial yield stress is almost absent between $300 \mathrm{~K}$ and $450 \mathrm{~K}$ and increases rapidly at higher temperatures. This can not be 
modelled with the common notion of the dynamic stress and therefore the dynamic stress is neglected here.

The work hardening part of the model, $\sigma_{\mathrm{w}}$, takes the evolution of the micro-structure into account. The relation between the dislocation density $\rho$ and $\sigma_{\mathrm{w}}$ is given by the Taylor equation:

$$
\sigma_{\mathrm{w}}=\alpha G(T) b \sqrt{\rho}
$$

where $\alpha$ is a scaling parameter of order 1 .

The essential part in these models is the evolution of the dislocation density. The creation and storage of dislocations is taken to be proportional to the mean free path, while dynamic recovery is taken to be proportional to the dislocation density itself. This leads to the basic equation for the Bergström model [7]:

$$
\frac{\mathrm{d} \rho}{\mathrm{d} \varepsilon}=c_{1} \frac{1}{L}-c_{2} \rho
$$

where the recovery parameter $c_{2}$ depends on temperature and strain rate.

In the original Bergström model, the mean free path $L$ was considered to be constant. The formation of dislocation walls and the principle of similitude led Vetter and van den Beukel [9], to a storage factor that is proportional to the square root of the dislocation density. The dynamic recovery term is considered to be due to annihilation and remobilisation of immobile dislocations. The remobilisation is a thermally activated process, based on vacancy climb [8]. The evolution of dislocation density is now reformulated as

$$
\frac{\mathrm{d} \rho}{\mathrm{d} \varepsilon}=U(\rho)-\Omega(\dot{\varepsilon}, T) \rho
$$

with

$$
\begin{aligned}
& U=U_{0} \sqrt{\rho} \\
& \Omega=\Omega_{0}+C \exp \left(-\frac{m Q_{\mathrm{v}}}{R T}\right) \dot{\varepsilon}^{-m}
\end{aligned}
$$

The function $U$ represents storage of mobile dislocations, and $\Omega$ represents dynamic recovery by remobilisation. The functions $U$ and especially $\Omega$ determine the shape of the hardening curve at different temperatures and strain rates. In the original model, $m$ was fixed at $1 / 3$, but here it is used as a fitting parameter. $Q_{\mathrm{v}}$ is an activation energy for vacancy migration.

Equation (10a) can be integrated analytically for constant $U_{0}$ and $\Omega$. For an incremental algorithm the dislocation density $\rho_{i+1}$ at time $t_{i+1}$ can be calculated from

$$
\rho_{i+1}=\left[\frac{U_{0}}{\Omega}\left(\exp \frac{\Omega \Delta \varepsilon}{2}-1\right)+\sqrt{\rho_{i}}\right]^{2} \exp (-\Omega \Delta \varepsilon)
$$

TABLE 1. Parameters for the Bergström model.

\begin{tabular}{llll}
\hline$\sigma_{0}$ & $109.3 \mathrm{MPa}$ & $m$ & 0.422 \\
$U_{0}$ & $6.093 \cdot 10^{8} \mathrm{~m}^{-1}$ & $G_{\text {ref }}$ & $26354 \mathrm{MPa}$ \\
$b$ & $2.857 \cdot 10^{-10} \mathrm{~m}$ & $\Omega_{0}$ & 23.63 \\
$C$ & $3.3422 \cdot 10^{5}$ & $Q_{\mathrm{v}}$ & $1.0917 \cdot 10^{5} \mathrm{~J} / \mathrm{mol}$ \\
$C_{\mathrm{T}}$ & 38.45 & $T_{1}$ & $2975 \mathrm{~K}$ \\
\hline
\end{tabular}

where $U_{0}$ and $\Omega$ are assumed to be constant during the time increment. For constant temperature and strain rate, substitution of (11) into (8) yields the Voce hardening equation. For non-constant temperature or strain rate, the two models differ. The Voce relation will result in an immediate stress change on a strain rate or temperature change, while the Bergström model is actually an evolution equation and, accordingly, the stress will only change gradually.

Finally the strain rate independent stress $\sigma_{0}(T)$ from Equation (7) must be determined. It is assumed that this stress is related to stresses in the atomic lattice. Hence, the temperature dependence of the shear modulus $G(T)$ is also used for $\sigma_{0}$. The flow stress is now evaluated by

$$
\sigma_{\mathrm{f}}=g(T)\left(\sigma_{0}+\alpha G_{\mathrm{ref}} b \sqrt{\rho}\right)
$$

where $g(T)$ is the shear modulus divided by the reference value $G_{\text {ref. }}$. The temperature dependence is numerically represented in this work by the empirical relation

$$
g(T)=1-C_{\mathrm{T}} \exp \left(-\frac{T_{1}}{T}\right)
$$

where $C_{\mathrm{T}}$ and $T_{1}$ are fitting parameters.

Some of the parameters in the Bergström model can be selected beforehand. The rest is determined by a least squares approximation of experimental tensile test results.

The initial dislocation density $\rho_{0}$ was chosen to be $10^{11} \mathrm{~m}^{-2}$, which seems to be a reasonable value for annealed aluminium. A ten times lower or higher value only had a small influence on the initial stages of plastic deformation. The magnitude of the Burgers vector $b$ and the shear modulus at room temperature $G_{\text {ref }}$ were taken from the literature and a value of $\alpha=1.0$ was chosen. The parameters $C_{\mathrm{T}}$ and $T_{1}$ could have been fitted to experimental values of the shear modulus, but better results were obtained by fitting them to the hardening curves, simultaneously with the other parameters.

The remaining parameters were fitted to tensile tests at two different strain rates and 4 different temperatures. It resulted in the values presented in Table 1. The RMS stress error for this fit is 4.30 which is significantly smaller than the 6.77 for the extended Nadai model.

In Figure 3 the simulated engineering stress-strain curves are plotted for the extended Nadai model and the Bergström model, together with the experimental data. It 


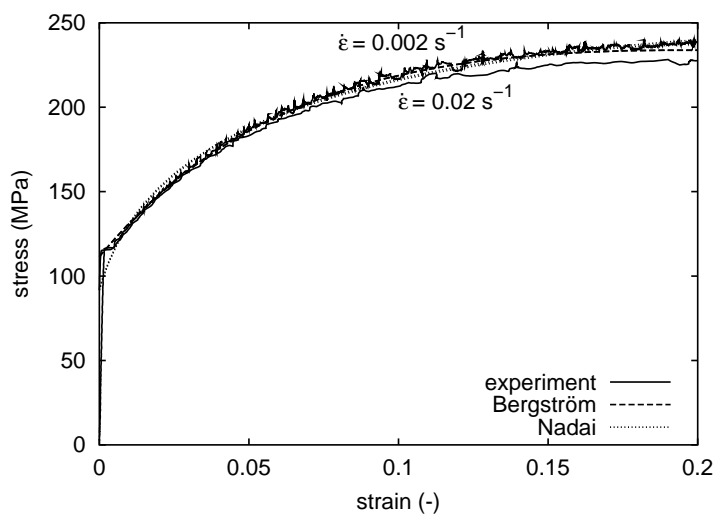

(a) $T=25^{\circ} \mathrm{C}$

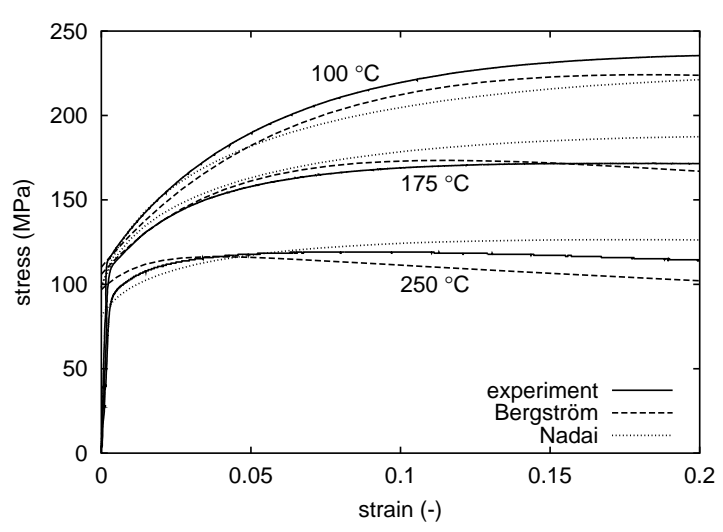

(b) $\dot{\varepsilon}=0.002 \mathrm{~s}^{-1}$

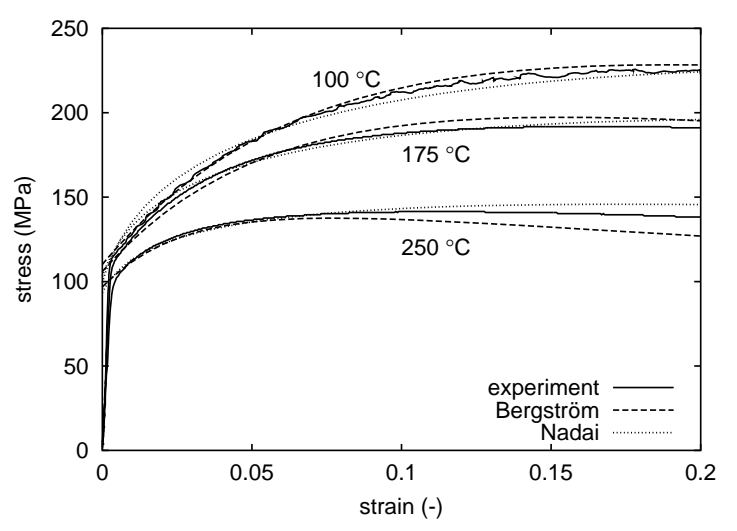

(c) $\dot{\varepsilon}=0.02 \mathrm{~s}^{-1}$

FIGURE 3. Engineering stress-strain curves - experiments and models. can be seen that both models are more or less capable of describing the experiments. It should be noted that the comparison is only valid for a uniform strain, which means up to the maximum engineering stress.

The stress-strain curves for temperatures of $100^{\circ} \mathrm{C}$, $175^{\circ} \mathrm{C}$ and $250^{\circ} \mathrm{C}$ are plotted in Figure 3(b) for a strain rate of $0.002 \mathrm{~s}^{-1}$ and in Figure 3(c) for a strain rate of $0.02 \mathrm{~s}^{-1}$. For the higher strain rate, both models perform quite well, specially for the strains below the stress maximum that were used in the parameter fitting. For the lower strain rate the differences are larger. The large stress drop between $100^{\circ} \mathrm{C}$ and $175^{\circ} \mathrm{C}$ is not very well represented by the extended Nadai model. For the extended Nadai model the flow stress at $100{ }^{\circ} \mathrm{C}$ is already too low, while the stress is still too high at $175^{\circ} \mathrm{C}$. The Bergström model does not perform very well if the initial yield stress is overestimated as in the low strain rate case at $250^{\circ} \mathrm{C}$.

\section{CYLINDRICAL CUP DEEP DRAWING}

Experiments were performed with a punch diameter of $110 \mathrm{~mm}$, punch radius $10 \mathrm{~mm}$, die hole diameter $113 \mathrm{~mm}$ and die shoulder radius $15 \mathrm{~mm}$. All experiments were performed with blanks of $230 \mathrm{~mm}$ diameter that were taken from an AA 5754-O sheet of $1.2 \mathrm{~mm}$ thickness. In the experiments, the effective punch stroke was $80 \mathrm{~mm}$ and the punch velocity $2 \mathrm{~mm} / \mathrm{s}$. The die and the blank holder were given a temperature of $25^{\circ} \mathrm{C}, 175^{\circ} \mathrm{C}$ and $250^{\circ} \mathrm{C}$, while the punch was kept at $25^{\circ} \mathrm{C}$. The blank holder force was equivalent to an initial pressure of 1.0 MPa on the contact area.

The friction between tool and workpiece is one of the least known factors in the simulations. For this combination of materials and a water-based lubricant, a friction coefficient of 0.06 was measured experimentally at room temperature. At temperatures above $150^{\circ} \mathrm{C}$, the friction coefficients varied between 0.12 and 0.18 in different experiments. It can be assumed that the increasing friction coefficient is due to the evaporation of water. Therefore, in the calculations a temperature dependent friction coefficient was used, with a linear relation from 0.06 to 0.12 for temperatures from $90^{\circ} \mathrm{C}$ to $110^{\circ} \mathrm{C}$ and constant before and after this range.

Orthotropic symmetry was assumed for the material model. A quarter of the blank was modelled and boundary conditions were applied on the displacement and rotation degrees of freedom to represent the symmetry. A relatively coarse finite element mesh was used with 934 discrete Kirchhoff triangular shell elements. The size of a typical element edge was $5 \mathrm{~mm}$.

To investigate the influence of the yield locus on the analysis results, simulations were performed with three 
TABLE 2. Equi-biaxial, plane strain and shear factors.

\begin{tabular}{cccc}
\hline & experimental & Von Mises & Hill '48 \\
\hline$f_{\mathrm{bi}}$ & 1.02 & 1.000 & 0.927 \\
$f_{\mathrm{ps}}$ & 1.15 & 1.155 & 1.101 \\
$f_{\mathrm{sh}}$ & 0.605 & 0.577 & 0.594 \\
\hline
\end{tabular}

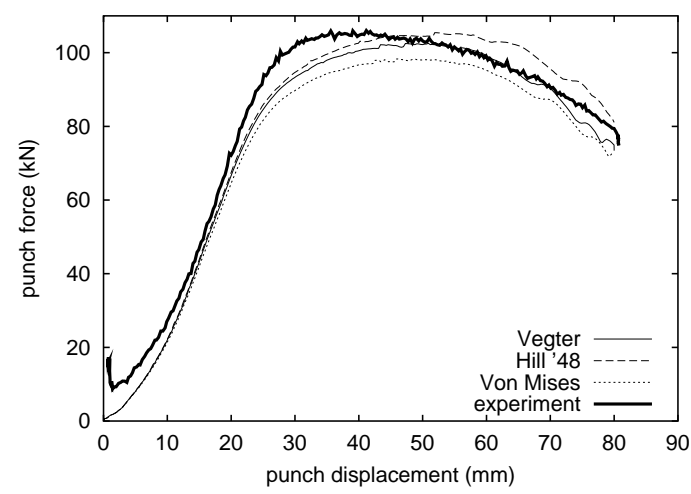

FIGURE 4. Punch force-displacement diagrams at $25^{\circ} \mathrm{C}$ with shell elements.

different yield loci. To exclude the influence of temperature and rate dependent material behaviour, a simple rate independent Nadai hardening model was used, representing the room temperature stress-strain curve. Analyses were run with a Von Mises, Hill ' 48 and Vegter material model. The same $R$-values were used for the Hill ' 48 and the Vegter models, namely $R_{0}=0.85, R_{45}=0.67$ and $R_{90}=0.70$. The Vegter model was directly fitted to the experimental biaxial stress ratios as given in Table 2 . For the friction coefficient a fixed value of 0.06 was used.

In Figure 4 it can be seen that the punch forcedisplacement curve for the Von Mises model is lower than for both other material models. This is attributed to the lower shear factor as presented in Table 2. The material deformation takes place mainly in the flange area, and in this area shear deformation dominates. Hence a lower shear factor will result in a lower punch force.

In Figure 5, the prediction of the wall thickness is presented. On the horizontal axis, the arc-length is given starting at the outer radius in the transverse direction, going to the centre, continuing to the outer radius in the rolling direction and returning to the transverse direction along the outer radius. All simulations predict too much thinning at the bottom of the cup, but the Hill ' 48 model performs notably badly. This can be attributed to the poor representation of the equi-biaxial stress by the Hill ' 48 model. Also for the Von Mises and Vegter model, the remaining difference with the measured thickness is still quite large and should be investigated further.

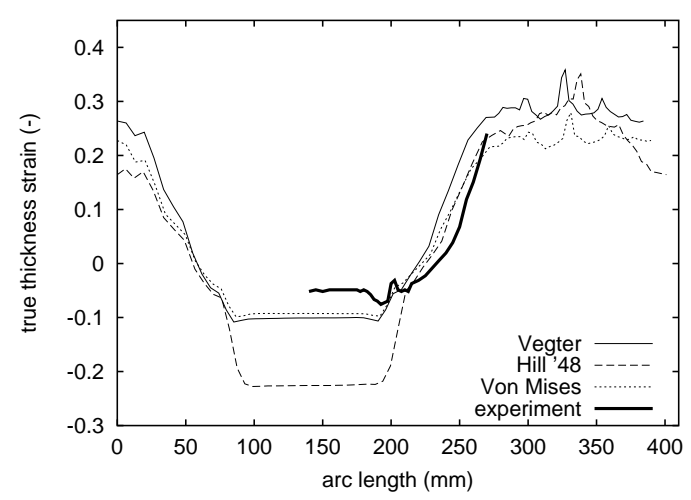

FIGURE 5. Thickness distributions at $25^{\circ} \mathrm{C}$ with shell elements.

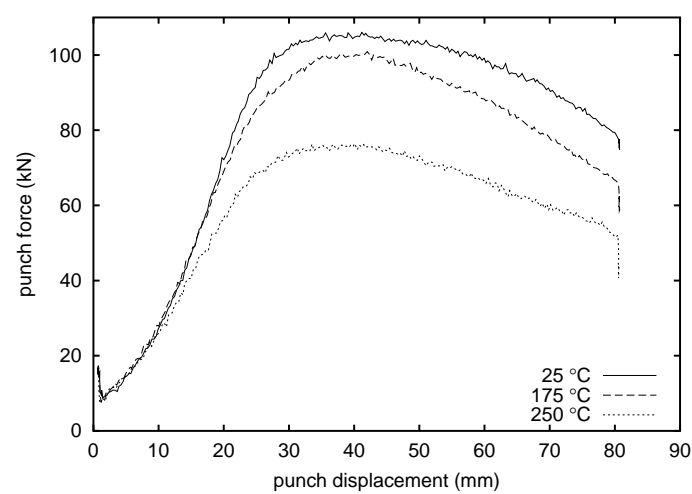

FIGURE 6. Punch force-displacement curves from experiments.

It should be noted here that not just the anisotropy, but specifically the shape of the yield locus is important. This is the origin of the so-called anomalous behaviour of aluminium. The stress in the bottom is mainly determined by the force needed to deform the flange area. In the flange area, the stress state can be characterised by the pure shear yield stress. Therefore, the ratio between equi-biaxial yield stress and pure shear yield stress is a better indicator for deep drawability than the commonly used $R$-value.

In Figure 6 and Figure 7 the experimental and predicted force-displacement curves from axisymmetric and shell element meshes are presented. In the axisymmetric models a Von Mises yield function is used and in the shell element models a Vegter yield function is used. For both sets of simulations, the temperature and rate dependent Bergström hardening model is used. The differences between the axisymmetric model and shell element model are relatively small. Compared to the experiments, the predicted force is a bit too low, but the general trends are predicted well. 


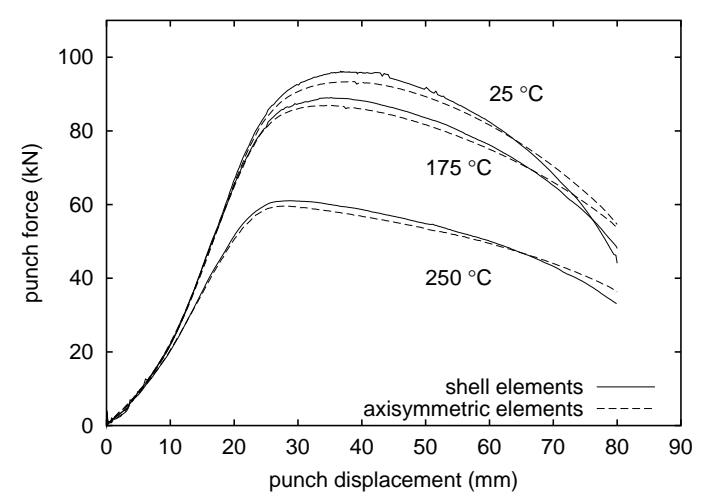

FIGURE 7. Punch force-displacement diagrams with axisymmetric and shell elements.

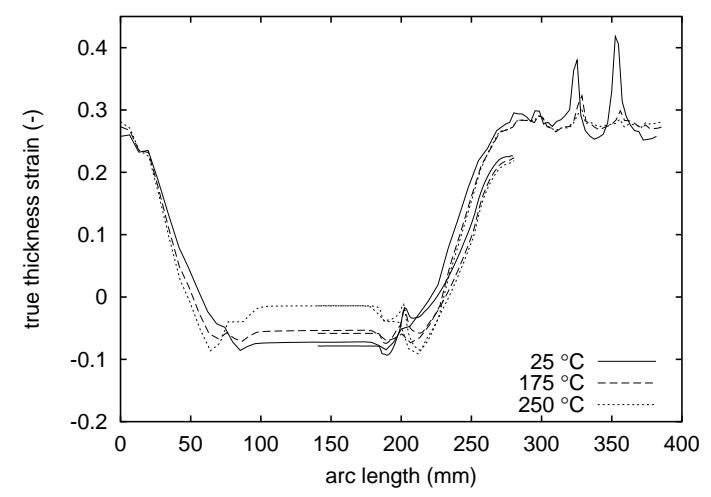

FIGURE 8. Thickness distributions with axisymmetric and shell elements (axisymmetric results start at $140 \mathrm{~mm}$ ).

\section{CONCLUSION}

The work hardening at different temperatures and strain rates was modelled by adapting the parameters of a Nadai model and with a physically based model. It was observed that the physically based Bergström model equals the phenomenological Voce model if the temperature and strain rate are constant. The temperature and strain rate sensitivity enters the Bergström model through a relatively simple modelling of dynamic recovery. It can predict stress-strain curves at constant strain rate very well, but the response on strain rate jumps is too slow. Also, the initial stress drop above $200{ }^{\circ} \mathrm{C}$ and low strain rate is underestimated.

More recent physically based models are extensions of the one-parameter models. Estrin [10] adapted the Kocks-Mecking model with a separate evolution of mobile and immobile dislocations. Results show a reasonable result for strain rate jumps, but at higher temperatures than used in warm forming. Nes [11], Roters et al. [12], Goerdeler and Gottstein [13] distinguish disloca- tion densities in cell walls and in cell interiors. The presented results are impressive, but the experimental validation seems to be focused on rolling of aluminium at higher temperatures than in warm forming. These advanced models are not considered here but are currently investigated on their applicability to warm forming. The distinction of stage II and stage III hardening in the Nes model may be helpfull in fixing the deviations for high temperature and low strain rate.

From the presented simulations of the cylindrical cup deep drawing, it can be concluded that the shape of the yield locus has an important effect on the calculated punch force-displacement curve and most notably on the predicted thickness distribution. The anisotropy in the material, modelled with the $R$-values, is less important than the ratios between equi-biaxial, plane strain, uniaxial and pure shear yield stresses. This was demonstrated by the large difference between the results for the Hill ' 48 and the Vegter models, that both used the same $R$-values.

\section{REFERENCES}

1. Wilson, D. V., Journal of Mechanical Working Technology, 16, 257-277 (1988).

2. Bolt, P. J., Lamboo, N. A. P. M., and Rozier, P. J. C. M., Journal of Materials Processing Technology, 115, 118-121 (2001).

3. Van den Boogaard, A. H., Bolt, P. J., and Werkhoven, R. J., International Journal of Forming Processes, 4, 361-375 (2001).

4. Van den Boogaard, A. H., Thermally enhanced forming of aluminium sheet-Modelling and experiments, Ph.D. thesis, University of Twente (2002).

5. Vegter, H., Drent, P., and Huétink, J., "A planar isotropic yield criterion based on mechanical testing at multi-axial stress states," in Simulation of Materials Processing: Theory, Methods and Applications, edited by S.-F. Shen and P. R. Dawson, Balkema, Rotterdam, 1995, pp. 345-350.

6. Vegter, H., and Van den Boogaard, A. H., International Journal of Plasticity (2004), submitted.

7. Bergström, Y., Materials Science and Engineering, 5, 193-200 (1969).

8. Bergström, Y., Reviews on Powder Metallurgy and Physical Ceramics, 2, 105-115 (1983).

9. Vetter, R., and van den Beukel, A., Scripta Metallurgica, 11, 143-146 (1977).

10. Estrin, Y., "Dislocation-Density-Related Constitutive Modeling," in Unified Constitutive Laws of Plastic Deformation, edited by A. S. Krausz and K. Krausz, Academic Press, San Diego, 1996, pp. 69-104, ISBN 0-12-425970-7.

11. Nes, E., Progress in Materials Science, 41, 129-193 (1998).

12. Roters, F., Raabe, D., and Gottstein, G., Acta Materialia, 48, 4181-4189 (2000).

13. Goerdeler, M., and Gottstein, G., Materials Science and Engineering A, 309-310, 377-381 (2001). 